Article original

\title{
L'impact des polymorphismes ABCB1 et CYP3A5 sur le cancer colorectal dans la population de l'Ouest Algérien: Etude cas-témoins
}

\author{
Effects of ABCB1 and CYP3A5 polymorphisms on colorectal cancer susceptibility in West \\ Algerian population: a case-control study
}
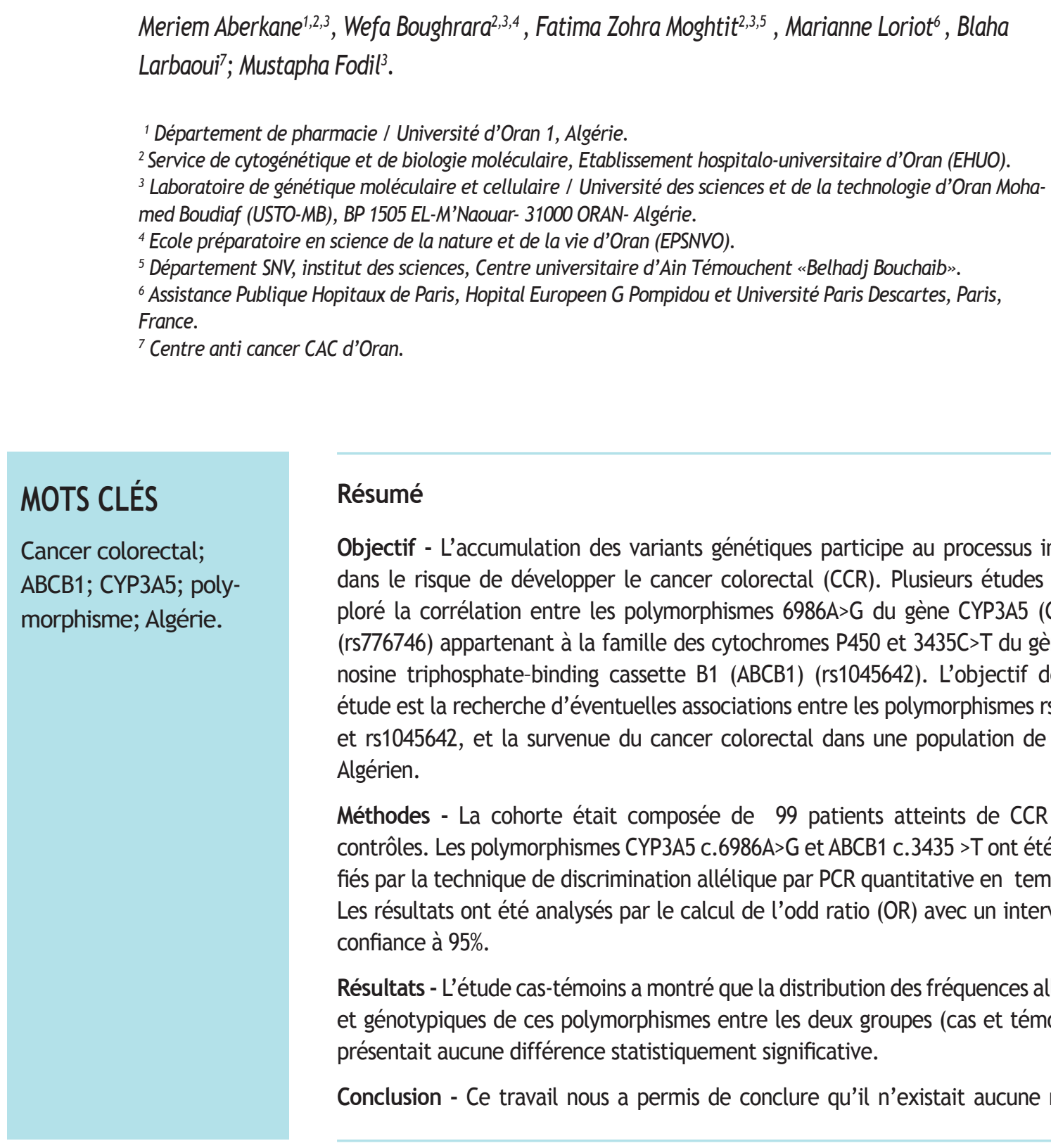

\section{Résumé}

Objectif - L'accumulation des variants génétiques participe au processus impliqué dans le risque de développer le cancer colorectal (CCR). Plusieurs études ont exploré la corrélation entre les polymorphismes 6986A>G du gène CYP3A5 (CYP450) (rs776746) appartenant à la famille des cytochromes $\mathrm{P} 450$ et $3435 \mathrm{C}>\mathrm{T}$ du gène adénosine triphosphate-binding cassette B1 (ABCB1) (rs1045642). L'objectif de cette étude est la recherche d'éventuelles associations entre les polymorphismes rs776746 et rs1045642, et la survenue du cancer colorectal dans une population de l'Ouest Algérien.

Méthodes - La cohorte était composée de 99 patients atteints de CCR et 101 contrôles. Les polymorphismes CYP3A5 c.6986A>G et ABCB1 c.3435 >T ont été identifiés par la technique de discrimination allélique par PCR quantitative en temps réel. Les résultats ont été analysés par le calcul de l'odd ratio (OR) avec un intervalle de confiance à $95 \%$.

Résultats - L'étude cas-témoins a montré que la distribution des fréquences alléliques et génotypiques de ces polymorphismes entre les deux groupes (cas et témoins) ne présentait aucune différence statistiquement significative.

Conclusion - Ce travail nous a permis de conclure qu'il n'existait aucune relation

${ }^{1}$ Auteur correspondant : aberkanemeriem@yahoo.fr 
d'association entre ces polymorphismes et la survenue du CCR dans notre population.

\section{KEY WORDS}

Colorectal cancer; ABCB1; CYP3A5; polymorphism; Algeria.

\section{Abstract}

Objective - The accumulation of genetic variants participates in the process involved in the risk of developing colorectal cancer (CRC). Several studies have explored the correlation between the 6986A> $G$ polymorphisms of the CYP3A5 (CYP450) gene (rs776746) belonging to the cytochrome P450 and 3435C> T family of the adenosine triphosphate-binding cassette B1 (ABCB1) gene (rs1045642).

The objective of this study is to investigate a possible association of rs 776746 and rs 1045642 polymorphisms with the occurrence of colorectal cancer on a population of western Algeria.

Methods - The cohort consisted of 99 CRC patients and 101 controls. The CYP3A5 c.6986A> $\mathrm{G}$ and $\mathrm{ABCB} 1$ c.3435> T polymorphisms were identified by the allelic discrimination by quantitative real-time PCR technique. The results were analyzed by calculating the odd ratio (OR) with a 95\% confidence interval.

Results - This case-control study didn't show any statistically significant difference in allelic and genotypic frequencies of these polymorphisms between the two groups (case and control).

Conclusion - This work allowed us to conclude that there was no association between these polymorphisms and the occurrence of CRC in our population.

\section{Introduction}

Le cancer colorectal (CCR) constitue un principal problème de santé publique dans le monde. En effet, il représente la deuxième cause de mortalité par cancer, avec 400.000 décès environ annuellement [1]. II est 10 fois plus fréquent aux Etats Unis d'Amérique qu'en Afrique [2]. Ces différences sont la conséquence du rôle essentiel de l'alimentation dans sa survenue [3]. En Europe, il représente le second cancer en terme de fréquence chez la femme, après le cancer du sein et le troisième chez l'homme après le cancer du poumon et celui de la prostate [4].

En Algérie et particulièrement à Oran, les cancers colorectaux sont en troisième position après ceux du poumon et de la vessie chez l'homme et ceux du sein et du col utérin chez la femme avec une augmentation de plus de $50 \%$ des cas entre 1986 et 2000 [5].

Les gènes considérés comme ayant une implication significative dans la mise en place du processus de cancérisation sont les oncogènes, les anti-oncogènes, les gènes de réparation de l'ADN et les gènes du métabolisme des xénobiotiques.

Les cytochromes P450 (CYP450) forment une super famille multi génique codant pour des enzymes de la phase I, im- pliquées dans le métabolisme oxydatif de diverses molécules aussi bien des xénobiotiques (médicaments, pesticides, polluants, cancérigènes...) que des substances endogènes (hormones stéroïdiennes, vitamines, acides gras...) [6].

Le polymorphisme CYP3A5*3C du gène CYP3A5 $(7 q 22,1)$ qui consiste en une transition 6986A>G (dbSNP Id : rs776746) dans l'intron 3 et qui crée un codon stop prématuré aboutissant à une absence d'activité de la protéine [7] a été associé à une diminution du risque de cancer de l'œsophage [8]. Cependant, à ce jour l'impact des polymorphismes génétiques de CYP3A5 sur le cancer colorectal n'a pas été clairement établi.

Le gène Adenosine Triphosphate-Binding Cassette B1 (ABCB1) $(7 q 21,1)$ qui code pour la protéine P-glycoprotéine (P-gp) [9] est exprimé de manière constitutive au niveau de certains organes notamment au niveau de l'intestin et des cellules endothéliales des capillaires sanguins de la barrière hémato-encéphalique. Etant donné le rôle physiologique de la P-gp, de nombreuses études ont pu mettre en évidence une association entre des polymorphismes altérant sa fonction et certains cancers comme la leucémie myéloïde chronique [10], le cancer du sein [11] et le cancer rénal [12]. Le polymorphisme le plus étudié est le polymorphisme ABCB1 c.3435 
C>T (dbSNP Id: rs1045642) localisé dans l'exon 26. Bien que ce dernier soit une mutation silencieuse codant pour l'isoleucine, ce polymorphisme semble affecter l'expression et la fonction de la P-gp de façon importante [13].

L'objectif de cette étude était donc de rechercher d'éventuelles associations entre les polymorphismes des gènes du métabolisme et du transport des xénobiotiques : CYP3A5 c.6986A $>G$ et $A B C B 1$ c. $3435 C>T$ et la survenue du CCR sur un échantillon de la population Ouest Algérienne.

\section{Matériels et Méthodes}

\section{Population d'étude}

Notre travail a été réalisé entre 2007 et 2012 sur une population $(n=200)$ constituée de 101 sujets sains non apparentés, ne présentant aucune maladie chronique (témoins), et de 99 cas atteints de cancer colorectal, admis au service d'oncologie du Centre Hospitalier Universitaire d'Oran et âgés entre 20 et 75ans,. Tous les individus recrutés dans cette étude sont originaires de l'Ouest Algérien. Chaque patient a donné son consentement éclairé pour participer à l'étude. Un questionnaire a été élaboré par les médecins pour chaque patient afin de recueillir les données démographiques et cliniques, les habitudes au tabagisme, la consommation d'alcool et les antécédents médicaux personnels et familiaux. Dans le groupe de contrôles, aucun individu n'avait d'antécédent de cancer. Environ 10 à $20 \mathrm{ml}$ d'échantillons de sang périphérique ont été recueillis dans des tubes d'Éthylène Diamine Tétra-Acétique (EDTA) et stockés à $-20^{\circ} \mathrm{C}$ jusqu'à l'analyse génétique.

\section{Extraction d'ADN}

L'ADN génomique a été isolé du sang périphérique en utilisant une technique standard de Miller, impliquant la digestion au dodécylsulfate de sodium (SDS)/protéinase K suivie d'une précipitation à l'éthanol [14].

\section{L'exploration des polymorphismes}

Les polymorphismes CYP3A5 c.6986A>G et ABCB1 c.3435C>T ont été identifiés par discrimination allélique par PCR quantitative en temps réel (Taqman, ABI PRISM® 7900 sequence detection system Applied Biosystems, Foster City, CA) [15]

en adaptant la chimie et les conditions recommandées par la biotechnologie Applied Biosystems.

Les sondes spécifiques utilisées ont été marquées à leur extrêmité 5' par les fluorophores FAM et VIC (Absolute Blue QPCR ROX Mix - Abgene ref AB- 4138/B) respectivement pour l'allèle 1 et l'allèle 2 de chacun des SNP (single nucleotid polymorphism) explorés.

Pour chaque polymorphisme, l'analyse a été réalisée en utilisant 1,5 미 d'ADN à $25 \mathrm{ng}$ dans un volume réactionnel Taqman final de 5 『l (5' nuclease Assay), composé de 2.5 미 du tampon « Taqman Genotyping Master Mix » (Applied Biosystems, Foster City, CA) à $2 \mathrm{X}$ et du mélange " sonde assay-specific » contenant les amorces spécifiques à chaque SNP et les sondes VIC et FAM à des concentrations définies par le biosystème d'analyse «Applied Biosystems ». Ces réactifs sont commandés en fonction de la référence $\mathrm{NCBI}$ « $\mathrm{rs}$ » (National Center for Biotechnology Information Reference) de chacun des variants et les conditions "Taqman " appliquées sont celles recommandées par le fabricant (Applied Biosystems : http://snp500cancer.nci.nih.gov/taqman_assays).

Une plaque de 384 puits a été utilisée et des contrôles homozygotes sauvages, homozygotes mutés et hétérozygotes correspondant à chacun des SNPs ont été ajoutés à la plaque. On obtient donc deux fluorescences pour un hétérozygote et une seule (VIC ou FAM) pour un homozygote.

Les séquences des amorces et des sondes Taqman utilisées pour l'analyse des polymorphismes sont représentées sur le (tableau 1).

\section{Analyse statistique}

La description statistique de l'échantillon testé a été effectuée et indiquée par une moyenne et une déviation standard ( \pm DS). Toutes les données statistiques ont été décrites en nombre et en fréquences. Les comparaisons des répartitions de fréquence des polymorphismes concernant les cas et les contrôles ont été effectuées à l'aide de tests X2 (programme Epi InfoTM version 7). Les valeurs de $p$ ont été considérées comme statistiquement significatives lorsque $p<0,05$; Le risque a été évalué en utilisant l'odd ratio (OR) et $95 \%$ d'in-

Tableau 1. Séquences des amorces et des sondes Taqman utilisées pour l'analyse des polymorphismes $A B C B 1$ c. 3435C>T, CYP3A5 c.6986A>G.

\begin{tabular}{|l|c|c|c|}
\hline Polymorphismes & Séquence des amorces & Sonde VIC \\
\hline $\begin{array}{l}\text { CYP3A5 c. } 6986 \mathrm{~A}>\mathrm{G} \\
\text { rs776746 }\end{array}$ & $\begin{array}{c}\text { Amorce F: CGAATGCTCTACTGTCATTTCTAACCA } \\
\text { Amorce R: TGAAGGGTAATGTGGTCCAAACAG }\end{array}$ & AGAGAGACTGAAAG & AGAGAGATTGAAAG \\
\hline $\begin{array}{l}\text { ABCB1 c. } 3435 \mathrm{C}>\mathrm{T} \\
\text { rs1045642 }\end{array}$ & $\begin{array}{l}\text { Amorce F: CTGTTTGACTGCAGCATTGCT } \\
\text { Amorce R: ATGTATGTTGGCCTCCTTTGCT }\end{array}$ & CCCTCACGATCTCTT & CCCTCACAATCTCTT \\
\hline
\end{tabular}


tervalle de confiance (IC).

\section{Résultats}

Nous avons étudié 99 patients Cancer colorectal et 101 témoins. Les caractéristiques de chaque groupe sont présentées dans le tableau 2.

Les résultats génotypiques pour le polymorphisme CYP3A5 C.6986A> $\mathrm{G}$ dans l'intron 3 du gène CYP3A5 ont été déterminés pour 96 patients atteints de CCR et 99 contrôles. Après comparaison des fréquences alléliques et génotypiques

Tableau 2. Distribution des sexes et âges entre cas et témoins.

\begin{tabular}{|l|c|c|}
\hline Variables & CCR & Témoins \\
& $n \quad(\%)$ & (\%) \\
Sexe & $(n=99)$ & $(n=101)$ \\
Homme & 42 & 31 \\
Femme & 30 & 17 \\
\hline Age (années) & & \\
$<50$ & 31 & 35 \\
$\geq 50$ & 41 & 13 \\
\hline
\end{tabular}

n:nombre, \%: pourcentage,SD: déviation standard.

du polymorphisme CYP3A5 c.6986A>G entre les cas et les contrôles, il est apparu qu'il n'existait aucune différence significative dans la distribution des fréquences des allèles CYP3A5*1 et CYP3A5*3 entre les deux catégories d'individus. Par ailleurs, les résultats génotypiques pour le polymorphisme $A B C B 1$ c. $3435 C>T$ ont été déterminés pour 94 patients atteints de CCR et 98 contrôles, Aucune différence statistiquement significative n'a été retrouvée pour le polymorphisme $A B C B 1$ c. $3435 C>T$ entre les patients $C C R$ et les contrôles.

\section{Discussion}

En Algérie, le cancer colorectal représente un réel problème de santé publique. Celui-ci combine l'implication de facteurs génétiques mais aussi environnementaux. De nombreux gènes sont associés au risque de survenue de cette pathologie dont certains codent pour des protéines intervenant dans le métabolisme et le transport des xénobiotiques.

\section{L'analyse du polymorphisme CYP3A5 c.6986A>G}

L'hypothèse qui justifie la présente étude est basée sur le fait que l'enzyme CYP3A5 est exprimée en grande quantité dans le tractus digestif et que son activité enzymatique est impliquée dans l'activation de plusieurs pro carcinogènes dont certaines mycotoxines comme l'aflatoxine B1 mais aussi les nitrosamines, les hydrocarbures aromatiques polycy- cliques, et les amines hétérocycliques produits au cours de la cuisson des aliments [16, 17]. Le SNP CYP3A5 6986A>G (dbSNP Id : rs776746) fait partie des polymorphismes récemment découverts qui aboutissent à des changements fonctionnels de cette enzyme [18]. Ce SNP donne naissance à une protéine tronquée, et nous l'avons exploré afin de définir la distribution de sa fréquence dans notre population d'une part et d'autre part pour rechercher une éventuelle association avec le risque de développement du CCR.

Nous avons tout d'abord établi une comparaison des fréquences alléliques de ce variant entre notre population témoin et d'autre population témoins à travers le monde. La fréquence de $80,6 \%$ de l'allèle CYP3A5*3 caractérisant notre population contrôle s'est révélée proche de celle retrouvée sur les populations Caucasienne (70\%) [19] et Japonaise (75\%) [20]. Aucune association avec le risque de CCR n'a été retrouvée après comparaison des fréquences alléliques et génotypiques du polymorphisme CYP3A5 c.6986A>G entre les cas et les contrôles. Sachant que l'allèle CYP3A5*3 est synonyme d'une expression plus faible voire nulle de l'enzyme CYP3A5, il semblerait que dans notre population, l'expression de CYP3A5 serait plus faible et donc que la perte de l'expression de cette enzyme due au polymorphisme CYP3A5 c.6986A>G n'ait pas de lien avec une susceptibilité accrue au CCR. Ceci est en accord avec les conclusions d'autres études [21, 22].

Cette absence d'association, malgré la fréquence élevée de ce SNP dans notre population, pourrait s'expliquer par des habitudes alimentaires différentes et donc une exposition à des substances cancérigènes potentielles différentes dans nos régions qui ne nécessiteraient pas la fonction enzymatique de CYP3A5 pour être désactivées. De plus, il se pourrait que l'expression de CYP3A5 ne soit pas exclusivement régulée au niveau de la transcription, et que les mécanismes de régulation de celle-ci puissent être différents entre les sujets souffrant de CCR et les individus sains [23]. Cette hypothèse signifierait que le polymorphisme modifiant la transcription du gène CYP3A5 pourrait être contrebalancé par un autre mécanisme de régulation qui aboutirait à une expression normale de cette enzyme chez les sujets malades. Par ailleurs, il serait tout à fait plausible de supposer que ce polymorphisme ait des conséquences différentes en fonction de l'alimentation et donc de la localisation géographique. En effet, ce polymorphisme est associé à une diminution du risque de cancer de l'œsophage mais aussi du cancer de la prostate $[24,25]$.

L'analyse du polymorphisme $A B C B 1$ c.3435 C>T Dans le groupe des sujets sains, la fréquence de l'allèle $A B C B 1$ $3435 C$ est largement supérieure à la fréquence de l'allèle ABCB1 3435T dans notre groupe de témoins (Tableau 03).

Cette même observation est également retrouvée dans les 
Tableau 3. Distribution des fréquences alléliques et génotypiques du polymorphisme CYP3A5 c.6986A>G chez des patients atteints de CCR et les contrôles de la population de l'Ouest Algérien.

\begin{tabular}{|c|c|c|c|c|}
\hline & $\begin{array}{c}\text { CCR } \\
\text { n (\%) }\end{array}$ & $\begin{array}{c}\text { Contrôles } \\
\text { n (\%) }\end{array}$ & $p$ value & OR (95\% TC) \\
\hline CYP3A5 c.6986A>G & $(n=96)$ & $(n=98)$ & & \\
\hline $\begin{array}{l}\text { Allèles } \\
\text { 6986A (CYP3A5*1) } \\
\text { 6986G (CYP3A5*3) }\end{array}$ & $\begin{array}{c}31(16,1) \\
161(83,8)\end{array}$ & $\begin{array}{c}40(20,4) \\
158(80,6)\end{array}$ & 0,4 & $\begin{array}{c}1^{b} \\
1,2[0,7-2,2]\end{array}$ \\
\hline $\begin{array}{l}\text { Génotypes } \\
\text { 6986AA (CYP3A5*1/*1) } \\
\text { 6986GA (CYP3A5*1/*3) } \\
\text { 6986GG (CYP3A5*3/*3) }\end{array}$ & $\begin{array}{c}1(1) \\
29(30,2) \\
66(68,7)\end{array}$ & $\begin{array}{c}6(6,1) \\
28(28,5) \\
65(66,3)\end{array}$ & $\begin{array}{l}0,1 \\
0,1\end{array}$ & $\begin{array}{c}1^{b} \\
0,1[0,003-1,4] \\
0,1[0,003-1,5]\end{array}$ \\
\hline
\end{tabular}

n:nombre, \%: pourcentage, OR: odds ratio, IC: intervalle de confiance, p: significativité, b: génotype / allèle catégorie de référence, CCR: cancer colorectale, a: teste de fisher.

populations Japonaise et Iranienne [26, 27] ce qui n'est pas le cas pour les populations Caucasiennes Espagnole et Américaine [28, 29]. Par ailleurs, la fréquence de ce SNP observée dans notre population se rapproche beaucoup de celle caractérisant la population Iranienne.

Les individus portant le génotype $3435 \mathrm{CC}$ présentent une expression de la P-gp duodénale 65 fois plus importante que les individus homozygotes 3435TT [13]. Cette surexpression de la protéine P-gp s'accompagne d'une résistance des sujets $3435 C$ C̀ l'entrée des xénobiotiques réduisant probablement ainsi le risque d'un CCR [9]. Dans cette étude, il apparait que le génotype $3435 C C$ n'est pas corrélé à la survenue du CCR dans la population de l'Ouest algérien (Tableau 4). Cette absence d'association est également retrouvée dans d'autres études cas/témoins [30].

Plusieurs facteurs tels que l'indice de masse corporelle (IMC) ou le statut de tabagisme peuvent influencer l'apparition du CCR; les polymorphismes génétiques ne sont donc pas les seuls facteurs impliqués dans la survenue de cette pathologie. La limitation la plus importante de notre étude pourrait être due à la petite taille de l'échantillon sachant qu'à mesure que la taille de l'échantillon augmente, la puissance augmente.

\section{Conclusion}

En conclusion, nos résultats représentent le premier rapport sur la population Algérienne démontrant l'absence d'association des polymorphismes CYP3A5 c.6986A>G et ABCB1 c.3435 C>T avec la survenue du cancer colorectal. Il serait peut-être plus intéressant d'étudier d'autres polymorphismes dans ces gènes qui pourraient être plus impliqués dans l'émergence de ce cancer.

\section{Remerciements}

Nous sommes reconnaissants aux patients atteints du CCR, à leurs familles, aux bénévoles sains et aux oncologues pour leur participation à cette étude.

Table 4. Distribution des fréquences alléliques et génotypiques du polymorphisme ABCB1 c. $3435 \mathrm{C}>\mathrm{T}$, chez des patients atteints de CCR et une population contrôle de l'Ouest Algérien

\begin{tabular}{|l|c|c|l|l|}
\hline & $\begin{array}{c}\text { CCR } \\
\mathrm{n}(\%)\end{array}$ & $\begin{array}{c}\text { Contrôles } \\
\mathrm{n}(\%)\end{array}$ & $p$ value & \multicolumn{1}{|c|}{ OR (95\% IC) } \\
\hline ABCB1 c.3435 C>T & $(n=94)$ & $(n=98)$ & & \\
\hline Allèles & & & & \\
$3435 \mathrm{C}$ & $121(64,3)$ & $129(65,8)$ & 0,8 & $1^{b}$ \\
$3435 T$ & $67(35,6)$ & $67(34,1)$ & & $0,9[0,6-1,4]$ \\
\hline Génotypes & & & & \\
$3435 C C$ & $39(41,4)$ & $44(44,8)$ & & \\
$3435 C T$ & $43(45,7)$ & $41(41,8)$ & 0,6 & $1^{b}$ \\
$3435 T T$ & $12(12,7)$ & $13(13,2)$ & 1 & $0,8[0,4-1,6]$ \\
\hline
\end{tabular}

n:nombre, \%: pourcentage, OR: odds ratio, IC: intervalle de confiance, p: significativité, b: génotype / allèle catégorie de référence, CCR: cancer colorectale, a: teste de Fisher. 
Nous remercions le $\operatorname{Pr}$ M.A Loriot et toute son équipe pour nous avoir aidés à mener cette étude.

\section{Conflits d'intérêt}

Tous les auteurs déclarent qu'ils n'existe aucun conflit d'intérêt.

\section{Références bibliographiques}

[1] European Journal of Cancer. 2005, $3: 291$.

[2] Jemal A, Siegel R, Ward E et al. Cancer statistics. CA Cancer J Clin 2006, 56:106-130.

[3] Irigaray P, Newby J A, Clapp R et al. Lifestylerelated factors and environmental agents causing cancer: An overview. Biomedicine \& Pharmacotherapy 2007; $6:$ 640-658.

[4] Boyle P, Ferlay J. Cancer incidence and mortality in Europe 2004. Ann Oncol 2005;16: 481-488.

[5] Registre du cancer d'Oran. 2006.

[6] Perera F.P. Environment and cancer: who are susceptible?. Science 1997, 278:1068-1073.

[7] Kuehl P, Zhang J, Lin Y et al. Sequence diversity in CYP3A promoters and characterization of the genetic basis of polymorphic CYP3A5 expression. Nat Genet 2001, 27(4):383-391.

[8] Dandara C, Ballo R; Parker M. CYP3A5 genotypes and risk of oesophageal cancer in two South African populations. Cancer Lett 2005, 225(2):275-282.

[9] Gottesman MM, Hrycyna CA, Schoenlein PV et al. Genetic analysis of the multidrug transporter. Annu Rev Genet 1995, 29:607-649.

[10] Urayama KY, Wiencke JK, Buffler PA et al. MDR1 gene variants, indoor insecticide exposure, and the risk of childhood acute lymphoblastic leukemia. Cancer Epidemiol Biomarkers Prev 2007, 16(6):1172-1177.

[11] Turgut S, Yaren A, Kursunluoglu R et al. MDR1 C3435T Polymorphism in Patients with Breast Cancer. Archives of Medical Research 2007, 38:539-544.

[12] Siegsmund M, Brinkmann U, Schaffeler E, et al. Association of the P-glycoprotein transporter MDR1(C3435T) polymorphism with the susceptibility to renal epithelial tumors. J Am Soc Nephrol 2002;13:1847-1854.

[13] Hoffmeyer S, Burk O, von Richter 0 et al. Functional polymorphisms of the human multidrugresistance gene: multiple sequence variations and correlation of one allele with Pglycoprotein expression and activity in vivo. Proc Natl Acad Sci USA 2000, 97:34733478.

[14] Miller S, Dykes D, Polesky H. A simple salting out procedure for extracting DNA from human nucleated cells. Nucleic Acids Research
1988, $16(3): 1215$.

[15] Livak K J, Marmaro J, Todd J A . Towards fully automated genome-wide polymorphism screening [letter]. Nat. Genet 1995, 9:341-342.

[16] Parke DV. The cytochromes P450 and mechanisms of chemical carcinogenesis. Environ Health Perspect 2005, 102:852-853.

[17] Windmill KF, McKinnon RA, Zhu X et al. The role of xenobiotic metabolizing enzymes in arylamine toxicity and carcinogenesis: functional and localization studies. Mutat Res 1997, 376:153-160.

[18] Van Schaik RH, Van der Heiden IP, Van den Anker JN et al. CYP3A5 variant allele frequencies in Dutch Caucasians. Clin Chem 2002, 48:1668-1671.

[19] Kurose K, Sugiyama E, Saito Y. Population differences in major functional polymorphisms of pharmacokinetic/pharmacodynamics-related genes in Eastern Asians and Europeans: implications in the clinical trials for novel drug development. Drug Metab. Pharmacokinet 2012, $27:$ 9- 54.

[20] Roy JN, Lajoie J, Zijenah L et (2005). Short communication: CYP3A5 genetic polymorphisms in different ethnic populations. Drug Metab. Dispos. 33, 884-887.

[21] Petrova DT, Yaramov N, Toshev S et al. Genotyping of CYP3A5 polymorphisms among Bulgarian patients with sporadic colorectal cancer and controls. Onkologie. 2007, 30(11):559-63.

[22] Gervasini G, García-Martín E, Ladero JM et al. Genetic variability in CYP3A4 and CYP3A5 in primary liver, gastric and colorectal cancer patients. BMC Cancer. 2007, 2 (7):118.

[23] Bergheim I, Bode C, Parlesak A. Decreased expression of cytochrome P450 protein in non-malignant colonic tissue of patients with colonic adenoma. BMC Gastroenterology 2005, 5:34.

[24] Dandara C, Ballo R, Parker M. CYP3A5 genotypes and risk of oesophageal cancer in two South African populations. Cancer Lett 2005, 225(2):275-82.

[25] Plummer S J, Conti D V, Paris P L et al. CYP3A4 and CYP3A5 genotypes, haplotypes, and risk of prostate cancer, Cancer Epidemiol. Biomarkers Prev. 12 (2003): 928-932.

[26] Komoto C, Nakamura T, Sakaeda T et al. MDR1 haplotype frequencies in Japanese and Caucasian, and in Japanese patients with colorectal cancer and esophageal cancer. Drug Metab Pharmacokinet 2006, 21(2):126-132

[27] Farnood A, Naderi N, Moghaddam SJ et al. The frequency of C3435T MDR1 gene polymorphism in Iranian patients with ulcerative colitis. Int J Colorectal Dis. 2007, 22(9):999-1003.

[28] Bernal ML, Sinues B, Fanlo A et al. Frequency distribution of C3435T mutation in exon 26 of the MDR1 gene in a Spanish population. Ther Drug Monit 2003, 25(1):107-111.

[29] Vicente J, Sinues B, Fanlo A et al. Polymorphism C3435T of the MDR1 gene in Central Americans and Spaniards. Mol Biol Rep 2007, 19. 
[30] Petrova DT, Nedeva P, Maslyankov $S$ et al. No association between MDR1 (ABCB1) 2677G>T and 3435C $>T$ polymorphism and sporadic colorectal cancer among Bulgarian patients. Cancer Res Clin Oncol 2008, 134(3):317- 322. 
